\title{
High-Q optical resonators: characterization and application to stabilization of lasers and high spectral purity microwave oscillators
}

\author{
O. Llopis ${ }^{\mathrm{a}, \mathrm{b}}$, P.H. Merrer ${ }^{\mathrm{a}, \mathrm{b}}$, A. Bouchier ${ }^{\mathrm{a}, \mathrm{b}}$, K. Saleh ${ }^{\mathrm{a}, \mathrm{b}}$, G. Cibiel $^{\mathrm{c}}$ \\ ${ }^{\mathrm{a} C N R S}$; LAAS ; 7 avenue du colonel Roche, F-31077 Toulouse, France \\ bUniversite de Toulouse ; UPS, INSA, INP, ISAE ; LAAS ; F-31077 Toulouse, France \\ ${ }^{\mathrm{c} C N E S}, 18$ avenue Edouard Belin, F-31401 Toulouse, France
}

Invited Paper

\begin{abstract}
Microwave optical systems for frequency generation are described in this paper. The goal is to reach high spectral purity in the microwave frequency range using ultra high $\mathrm{Q}$ optical resonators. The resonators investigated are of two types : resonant (passive) fiber rings and WGM tridimensional resonators. They all feature ultra high optical Q factors, in excess of $10^{8}$ or $10^{9}$ near $1550 \mathrm{~nm}$. These resonators also sustain a large number of optical resonances, and the microwave signal is stabilized on two (or more) resonances of this optical comb. Different problems have to be overcome in order to reach a functional system, such as : resonator design and coupling, laser stabilization on a resonance, overall system design, noise optimization... This paper gives an overlook on these problems, and on some solutions we found to work towards a compact and efficient microwave opto-electronic oscillator (OEO). A first result is presented on a $10 \mathrm{GHz}$ OEO based on a resonant fiber ring.
\end{abstract}

Keywords: microwave oscillator, optical resonator, WGM resonator, phase noise, optical fiber

\section{INTRODUCTION}

The field of high spectral purity microwave oscillators is today reaching a limit in terms of quality factor and phase noise. Firstly, CAD modeling approaches, together with the improvement of the transistors technology, have greatly reduced the phase noise generated by the active devices in the oscillating loop. Secondly, for the frequency reference device, the mostly used high Q microwave resonators are dielectric resonators, which have been studied for many years now. The highest performances are obtained with whispering gallery modes sapphire resonators, which take benefit of the ultra low dielectric losses of monocristaline sapphire at microwaves. Q factors in the range of $10^{5}$ at $10 \mathrm{GHz}$ are obtained with this technique, but the relatively large size of these resonators when packaged (about $10 \mathrm{~cm}$ in diameter and $5 \mathrm{~cm}$ in height) restrict the use of these oscillators to metrology applications. Moreover, the microwave loss tangent of dielectric materials is generally inversely proportional to the frequency, which means that if the design of microwave oscillators at higher frequencies (millimeter wave range) is a good solution to reduce the resonator size, it should not solve the problem of the limits in terms of $\mathrm{Q}$ factor.

A solution to overcome these limitations is to carry the microwave signal on another wave, such as an acoustic wave or an optical wave. The case of the acoustic wave is today investigated in bulk acoustic wave resonators using thin films techniques, for which the small size allows a resonance at microwave frequencies. This approach leads to small (about $100 \mu \mathrm{m}^{2}$ ) and efficient resonators, post-processed on silicon circuits (above IC approach), which focus the wireless market. However these resonators are limited both in their operating frequency (today about $5 \mathrm{GHz}$ ) and their Q factor values (in the range of 500). The case of optics is different : what we are looking for is very high performance at higher frequencies. The operation principle uses an optical carrier, delivered by a laser, a modulator, to transpose the microwave signal around the optical carrier, an optical frequency reference device (resonator or delay line) and a fast photodiode to bring back the signal to microwaves. 
The first opto-electronic oscillators (OEO) were based on optical delay lines [1,2]. The equivalent $\mathrm{Q}$ factor of a delay line can be calculated from its phase to frequency slope [3] and, as an example, a $4 \mathrm{~km}$ optical delay line features a delay of $19 \mu \mathrm{s}$ and an equivalent Q factor higher than $10^{6}$ at $20 \mathrm{GHz}$. Very high performance microwave oscillators have been designed with this approach [2,4]. However, these oscillators suffer from two difficult to solve problems : 1) they are quite bulky, because of the fiber spool ; 2) their phase noise spectrum features many parasitic modes due to the large number of frequencies for which a $2 \pi$ phase condition is realized in these devices. In order to solve the second problem, different techniques have been proposed (such as using two or more spools matching at only one frequency), but these techniques are not always efficient enough because of the high density of parasitic modes ; they also increase the size of a system which is already big enough.

An alternative approach to the optical fiber delay line for the OEO has been proposed as early as 1999 [5]. This approach consists in replacing the delay line by a high Q optical resonator, in order to reduce the size of the device. This idea looks afterwards quite obvious, but the difficulties to realize the whole system with an optical resonator are numerous and have up to now prevented the design of a very high performance system with this approach. However, in the authors opinion, the potential for a real breakthrough in microwave frequency generation lies today in these systems, because very high optical $Q$ factors have been obtained, in excess of $10^{9}$ or $10^{10}$, using relatively small devices. Moreover, the availability of stable microwave frequency combs using these resonators is particularly interesting for many applications, where these devices will work together with an atomic frequency reference.

Up to now, three types of OEO based on a resonant device have been proposed : 1) OEOs based on a WGM micro or mini-disk resonator [5 to 8] ; 2) OEOs using a fiber laser which includes a relatively long fiber resonant loop shared by the RF loop [9], called COEO for Coupled Opto-electric Oscillators ; 3) OEOs using a passive fiber ring resonator [10]. In each of these cases, many different approaches are possible for the resonator itself or the system topology.

For the resonator, the best $\mathrm{Q}$ factors have been obtained in monocristaline polished disk resonators, with $\mathrm{Q}$ factors as high as $10^{11}$ with $\mathrm{CaF}_{2}$ material [11]. However, the use of these 3D resonators is difficult, mainly because of the lack of integration techniques which include the coupling to the fiber. The case of the COEO is particularly interesting, because the laser is naturally stabilized on the resonator (the resonator is part of the optical source). It has to be compared in terms of performances ( $\mathrm{Q}$ factor, phase noise) to the last case, which uses a passive fiber ring which features an ultra high $\mathrm{Q}$ factor with, however, the need to realize a feedback to stabilize the laser on the resonator.

In this paper, we will summarize the work performed in this field at LAAS Toulouse these last years. Our approach includes investigations on both 3D WGM resonators and on fiber rings resonators. It also includes investigations on various techniques for the laser stabilization onto the resonator, and on the development of specific characterization tools to extract the resonator main parameters. Finally, a preliminary OEO system has been designed and studied, both experimentally and theoretically. The performances of this OEO are still modest, but we will explain the different path we are following today to improve its phase noise.

\section{HIGH Q OPTICAL RESONATORS}

We started the investigation on high Q optical resonators for microwave frequency generation by the characterization of silica microspheres realized at ENSSAT Lannion (France). A measurement bench has been set up to characterize the optical properties of these resonators (frequency comb, quality factor) using tapered and half tapered fibers (Figure 1) for the coupling. However, the small size of these resonators (400 $\mu \mathrm{m}$ in diameter), and the resulting large FSR (above $100 \mathrm{GHz}$ ), makes the use of these spheres difficult for microwave filtering. Then, larger silica spheres (Ø $3.3 \mathrm{~mm})$ have been realized using a $\mathrm{CO}_{2}$ laser. These spheres feature a Q factor of about $210^{8}$ together with a FSR of $20 \mathrm{GHz}$ [12] compatible with many microwave applications and with currently available optical telecommunications devices (modulators, photodiodes...). In order to increase the Q factor, we have also investigated, like other research groups [10 to 14], monocristalline disk resonators. Two resonators of about $7 \mathrm{~mm}$ in diameter have been realized by polishing on the edge a quartz disk and a $\mathrm{CaF}_{2}$ disk. As shown later in this paper, a $\mathrm{Q}$ factor value of $410^{9}$ has been observed on the quartz disk while some polishing problems have greatly reduced the performance of the $\mathrm{CaF}_{2}$ disk (a new $\mathrm{CaF}_{2}$ disk is under test at this time).

The main problem with these resonators lies in the coupling techniques. Indeed, even if the coupling with tapered fibers is very efficient, it is difficult to set up for large devices such as mm spheres or disks (need for a very long taper) and, moreover, this type of coupling approach cannot be integrated (it is a laboratory approach only). Other techniques 
use either a prism or angle polished fibers. In the case of angle polished fiber, the coupling to high index materials is not possible, and the technique is limited to silica spheres or $\mathrm{CaF}_{2}$ disks. In any case, the distance between the coupling device and the resonator has to be controlled with an extreme precision.

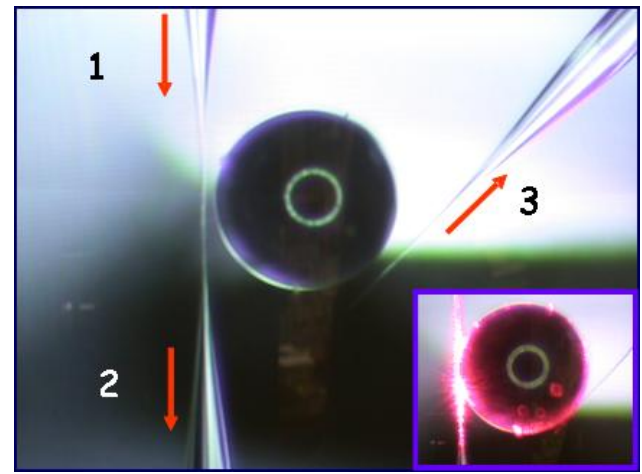

Figure 1. Coupling of a silica microsphere with tapered fibers (inset : coupling check with red laser)

Up to now, practical applications with these resonators are facing the problem of the integration (or assembly) of these coupling elements. However, another type of resonator can reach such high Q factors : the fiber ring resonator [15]. The size of fiber ring resonators is of course larger than the size of 3D resonators, but these resonators are quasi planar structures which can be easily integrated in (or close to) the microwave-optical circuit substrate, providing the size of the circuit is large enough to host the fiber loops (about $10 \mathrm{~cm}$ in diameter). Such a resonator is presented in Figure 2. The Q factor of these resonators is dependent on the additional losses in the fiber couplers, in the fiber splices and, above all, on the loop length. As an example, a loop length in the range of $20 \mathrm{~m}$ allows to reach a Q factor of $310^{9}$ (measured), together with an FSR of about $10 \mathrm{MHz}$ [3]. Such a Q factor is particularly interesting for OEO applications.

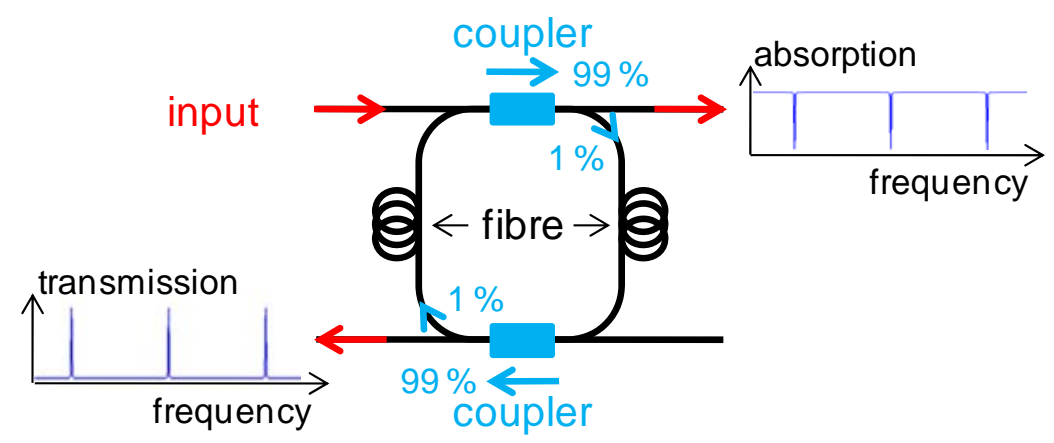

Figure 2. Example of a fiber ring resonator, with resonant modes in absorption and in transmission

\section{RESONATORS CHARACTERIZATION}

The first step in OEO studies is to set up a characterization bench for the resonators to be used in the OEO. This is not easy, because of the coupling problems explained above, and because of the extremely small resonance bandwidth to be characterized.

A dedicated measurement bench has thus been set up, using three axis piezoelectric nanometric control the two resonator access (in our applications, the resonators are, up to now, mainly used in transmission mode). This measurement bench has been built on an optical table and is entirely protected from dust. This allows us to get an efficient coupling with tapered fibers and, in some cases, with angle polished fibers. 
The extremely high $\mathrm{Q}$ does not allow inducing in these resonators a sufficient amount of light from conventional lasers. As an example, a $\mathrm{Q}$ factor of $10^{9}$ near $\lambda=1550 \mathrm{~nm}$ results in a $3 \mathrm{~dB}$ bandwidth of $200 \mathrm{kHz}$, which is lower than the spectral width of semiconductor lasers. A first solution is to use a high spectral purity laser (such as a fiber laser), but this is only one part of the solution because when the light is induced in the resonator, the resonator is heated by this light (even at low power, because of the lifetime of the photons in such resonators), the resonator frequency is thus shifted and the signal is lost again.

Taking into account these problems, three techniques have been investigated and compared one to another to measure the resonator half bandwidth. The first one is the optical scan method, which uses the slow tuning of a high spectral purity laser to scan the resonant modes. The second one is the cavity ring down method, which has been set up at ENSSAT Lannion, and which is a time domain approach. The third one is a measurement based on a microwave network analyzer, so in the spectral domain again, which features an extremely high spectral resolution.

\section{1) The scan method}

The method consists in scanning the laser frequency to explore the frequency comb. The determination of the FSR is thus direct if the laser tuning range is large enough. For small size resonators, the FSR can be highest than $10 \mathrm{GHz}$. In our case, we use an amplified erbium-doped fibre laser from Koheras. It is tunable on a frequency range of $120 \mathrm{GHz}$ thanks to the variation of its temperature. The second point is the measurement of the full-width at half-maximum of a resonant mode. This parameter leads to the finesse and the quality factor of the resonator. Our Koheras laser presents a really low linewidth of about $1 \mathrm{kHz}$. We can thus measure with a fairly good resolution the main resonant modes. Apart from the temperature control, this laser can be tuned faster on a range of $2 \mathrm{GHz}$ with a piezoelectric component. It is thus possible to observe the mode width precisely, as shown in Figure 3 in the case of the 7.7 mm quartz minidisk in absorption mode. For this resonator, and with this technique, a half bandwidth of $45 \mathrm{kHz}$ has been measured on a single access device, which leads to a Q factor higher than $410^{9}$.

A good estimate of the resonator Q factor is provided by this scan technique. However, because of the problem of the resonant frequency shift during the measurement, the scan velocity should be carefully adjusted for each type of resonator, and it is difficult to be sure that the observed resonant curve is completely free from this problem. Therefore, more precise techniques have to be set-up to precisely characterize the resonator half-bandwidth.

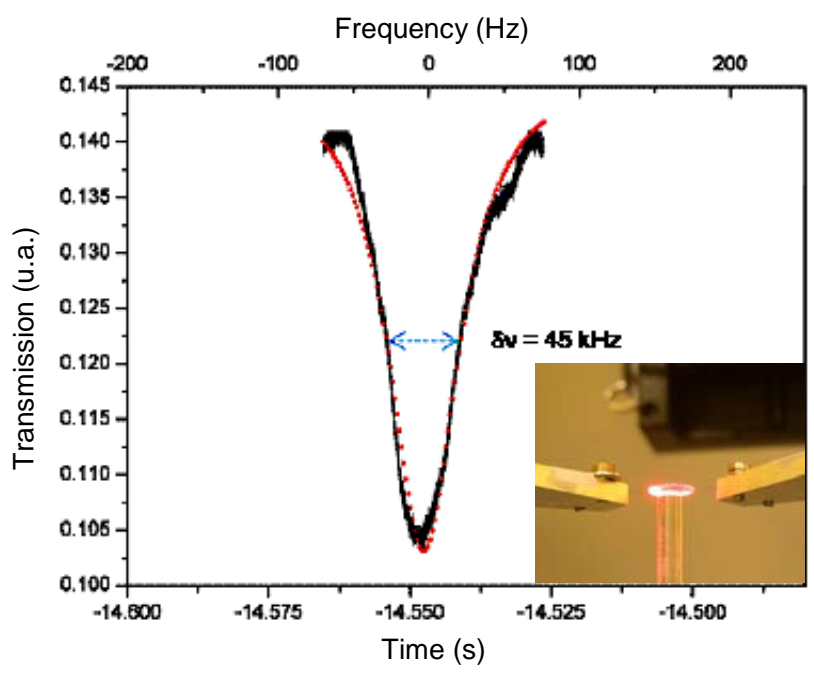

Figure 3. Scan measurement of a $7.7 \mathrm{~mm}$ mini-disk resonator made of polished quartz material

\section{2) The cavity ring down (CRD) method}

The cavity ring down method avoids the difficulty of the comparison between the laser linewidth and the resonant mode, because the measurement occurs directly in the time domain. The principle is the same than for the scan method, 
but the laser frequency is quickly tuned contrarily to the previous method. For the CRD method, the thermal effects do not have time to appear and the mode is described with as less perturbation as possible. The aim is to measure the photon lifetime in the resonant cavity by studying its relaxation regime. With a simulation of the CRD analysis of the cavity [16], it is possible to determine the different contributions for the optical quality factor: intrinsic factor, linked to the internal losses of the cavity, the coupling factor, linked to the couplers characteristics and the global factor, which characterizes the resonator in the system. It also shows the coupling regime of the resonator: critical, under-coupled or over-coupled. An example of the measurement that can be obtained with a fibre ring resonator is shown in Figure 4 . This measurement has been performed at ENSSAT Lannion (France).

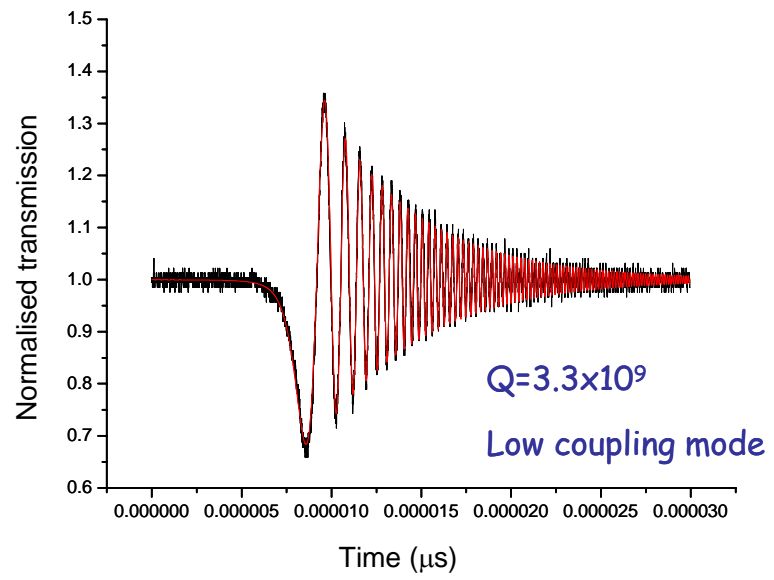

Figure 4. CRD measurement of a 20m-long fibre ring resonator (ENSSAT, Lannion).

\section{3) RF or Microwave measurement of the optical resonances}

Our final application is at microwave frequencies. It is therefore essential to test the resonator behaviour in a configuration which is as close as possible from the OEO system. The test configuration we have developed was clearly dedicated to OEO design, but it is also very efficient to measure the optical properties of the resonators with a precision in frequency which cannot be obtained with all optical techniques.

The main problem was to get rid of the frequency drift due to the resonator self eating. The solution to this problem is to lock the laser onto the resonator frequency. This has been realized using a Pound-Drever feedback loop [12,15]. A schematic of the experimental setup is shown in Figure 5.

The validity of this approach has been verified experimentally on different fiber ring resonators. When the PoundDrever loop is closed, the resonator transmission is locked to its maximum value, ie the laser is locked onto one of the resonances. If the controller gain and integration time of the proportional integrator differentiator (PID) have been correctly chosen, the correction maintains the laser locked onto the resonance for a long time. As soon as the laser is locked onto the resonance, it is possible to use the system for microwave applications. A microwave optical amplitude modulator (Mach-Zehnder modulator) is added to the system. The signal coming out from a microwave network analyser feeds the modulator, goes through the optical resonator and is finally recovered on a fast photodiode and analysed on port 2 of the network analyser.

The optical frequency comb of the resonator can then be measured, and a focus on any mode gives the information on the resonator half bandwidth and Q factor with the precision of the microwave network analyser, which is as good as $0.1 \mathrm{~Hz}$. Any problem on the resonance shape is immediately shown by this experiment (such as two modes competing each other), and the only limit in precision is in the ability of locking the laser onto one of the resonances. Figure 5 shows an example of such a measurement performed on a $20 \mathrm{~m}$ fibre ring resonator. The measured $\mathrm{Q}$ factor is very close to the one obtained from the CRD experiment. 


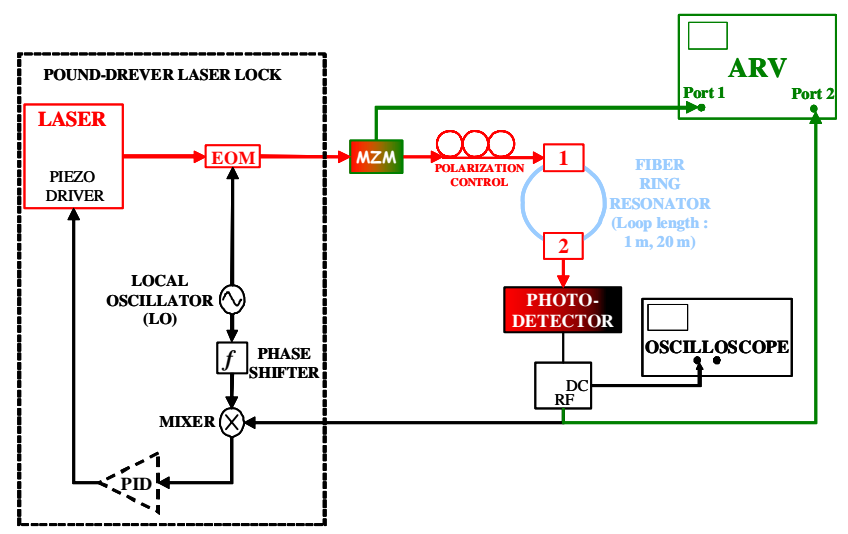

Figure 5. Stabilization of the Koheras laser on the high Q resonator thanks to the Pound Drever Hall approach, and measurement of the frequency response of the resonator using a microwave network analyser

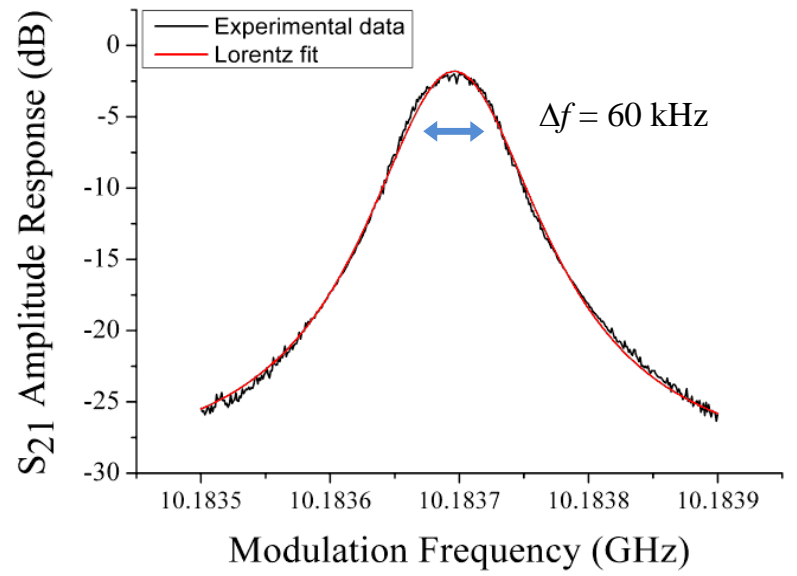

Figure 6. Measurement of a $20 \mathrm{~m}$ fiber ring resonator using the microwave approach (from ref. [3])

\section{MICROWAVE OSCILLATOR BASED ON AN OPTICAL RESONATOR}

If we are able to filter a microwave signal, as demonstrated in Figure 6, we may use the same approach to stabilize a microwave oscillator. The OEO set-up is depicted in Figure 7. This set up includes the optical resonator and two different feedback loops : one low frequency feedback loop to stabilize the laser on one mode of the resonator, and a microwave feedback loop to start an oscillation on one of the lateral side-modes of the resonator frequency comb. The microwave loop includes two microwave amplifiers and a phase shifter, in order to adjust the conditions for the starting up of the oscillation (unity gain higher than one and zero loop phase at the oscillation frequency).

The only problem to start an oscillation on such an optical resonance is due to the frequency comb, particularly if the resonator FSR is small. This is the case for relatively large fiber ring resonators. As an example, the $20 \mathrm{~m}$ ring resonator presented here features a FSR of $10 \mathrm{MHz}$, which makes possible the growing up of an oscillation on a large number of frequencies separated each other from $10 \mathrm{MHz}$. To isolate one of these frequencies, a conventional microwave dielectric 10.2 GHz resonator has been added in the RF feedback loop. The frequency of this resonator is tuned to the frequency of one of the modes of the optical resonator thanks to a tuning screw above the microwave resonator, and the lateral sidemodes are thus rejected, as shown in Figure 8. 


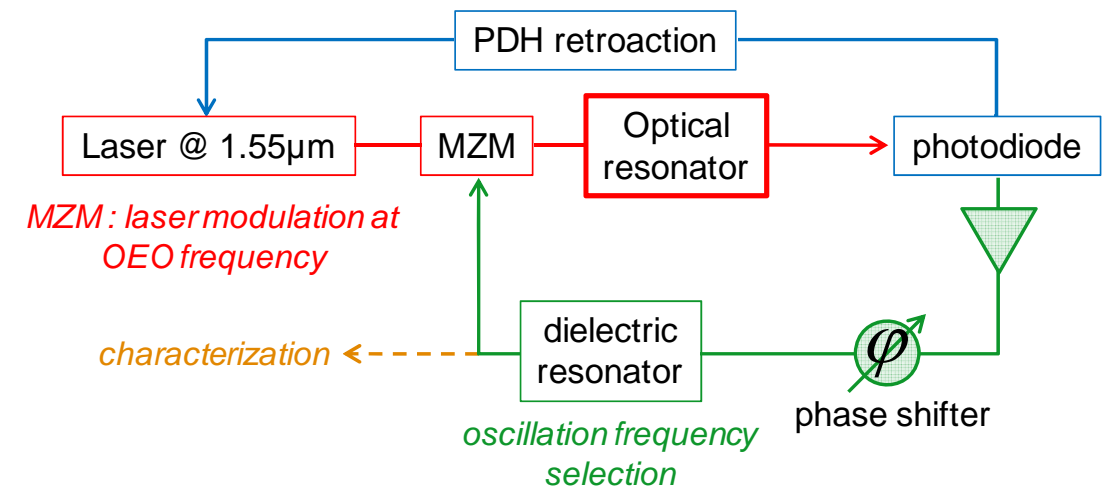

Figure 7. Schematic of the $10 \mathrm{GHz}$ OEO stabilized on a fiber ring resonator. The Pound-Drever-Hall (PDH) low frequency feedback stabilizes the laser and the oscillation starts thanks to the microwave feedback loop.

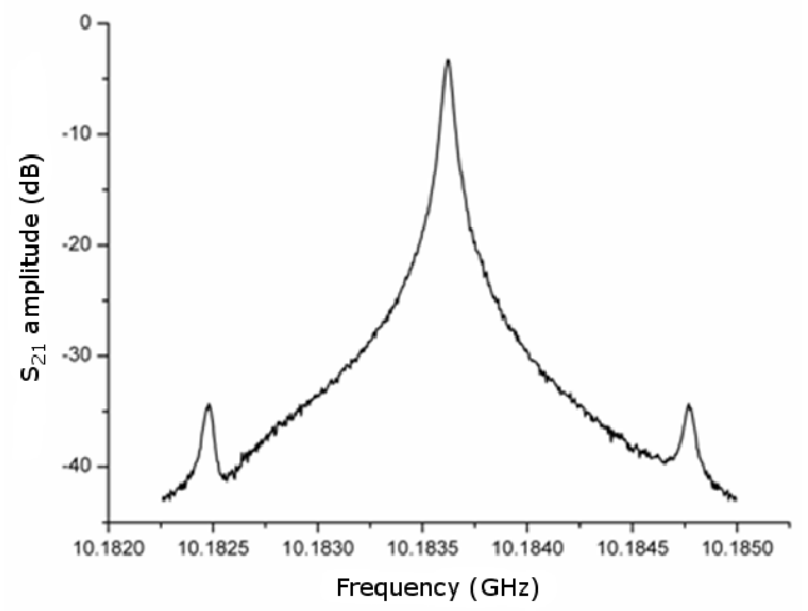

Figure 8. Measurement of the resonance of the $20 \mathrm{~m}$ ring resonator after filtering by the microwave dielectric resonator The two first lateral side-modes are rejected of more than $30 \mathrm{~dB}$

In spite of an ultra high Q factor of about $1.510^{5}$ at $10 \mathrm{GHz}\left(310^{9}\right.$ at $1550 \mathrm{~nm}$, or $200 \mathrm{THz}$ ), the first oscillator realized with this approach has revealed a relatively high level of $1 / \mathrm{f}$ frequency noise. This frequency noise component was apparently generated by the resonator itself, and a new $10 \mathrm{~m}$ fibre ring resonator which solves this problem has thus been developed (patent pending). However, this new resonator was effectively less noisy but was also featuring a lower optical quality factor, of around $210^{8}$. Despite this relatively low Q, a first oscillator has been assembled with this new resonator [10]. The spectrum of this oscillator and its phase noise spectrum, measured thanks to an Agilent E5052B signal analyzer, are presented in Fig. 9.

The phase noise spectrum features different slopes. Between $10 \mathrm{~Hz}$ and $1 \mathrm{kHz}$, the phase noise is dominated by the $1 / \mathrm{f}$ frequency noise, which is characterized by a slope of $-30 \mathrm{~dB} / \mathrm{dec}$. The white frequency noise is characterized by a slope of $-20 \mathrm{~dB} / \mathrm{dec}$ from 1 to $400 \mathrm{kHz}$ and is related to the carrier to noise ratio in the RF oscillating loop, and more precisely to the carrier to noise ratio at the output of the photodiode (CNR of the optical link). The phase noise floor appears after $400 \mathrm{kHz}$, and has a value of about $-122 \mathrm{dBc} / \mathrm{Hz}$. The final decrease of the noise is due to the filtering by the dielectric resonator. The frequency $f_{c}$ at the frontier between white frequency noise and the phase noise floor is related to the loaded microwave quality factor $\left(Q_{R F}\right)$ and the microwave frequency $f_{R F}$ by the relation $f_{c}=f_{R F} /\left(2 Q_{R F}\right)$ through Leeson's simplified model of feedback oscillators [17]. With a corner frequency of $0.4 \mathrm{MHz}$, the loaded microwave quality factor is $1.210^{4}$ at $10 \mathrm{GHz}$, which is in good agreement with an optical quality factor of $210^{8}$. 
In spite of its good quality factor, this oscillator presents a relatively high phase noise level. For the white frequency noise region $(20 \mathrm{~dB} / \mathrm{dec})$, this is mainly due to the transmission losses in the optical device. These losses contribute to decrease the signal to noise ratio at the output of the resonator, and thus to increase the phase noise of the oscillator. For the $1 / \mathrm{f}$ frequency noise region $(30 \mathrm{~dB} / \mathrm{dec})$, the components of this system, either electronic or optoelectronic, have not been optimized versus noise and this will be done in next OEOs. To this purpose, we have developed an original CAD modelling approach of microwave-optical systems which includes the noise modelling [18], and more precisely the three noise contributions to these systems : microwave noise, low frequency (1/f) noise and optical noise. This approach is based on a microwave CAD software, Agilent ADS, which is able to compute the noise conversions between different carriers thanks to a frequency domain approach of non-linear systems (harmonic balance). With this technique, the optical carrier is considered just like a microwave carrier, with its frequency and amplitude noise components, and the other optical or optoelectronic elements of the system are described either by electronic equivalent circuits or by using mathematical nonlinear black boxes. Up to now, we are able to compute the noise in an open loop microwave optical system with this approach. The case of autonomous circuits (such as the OEO) is a bit more complex, but we are currently working on it.

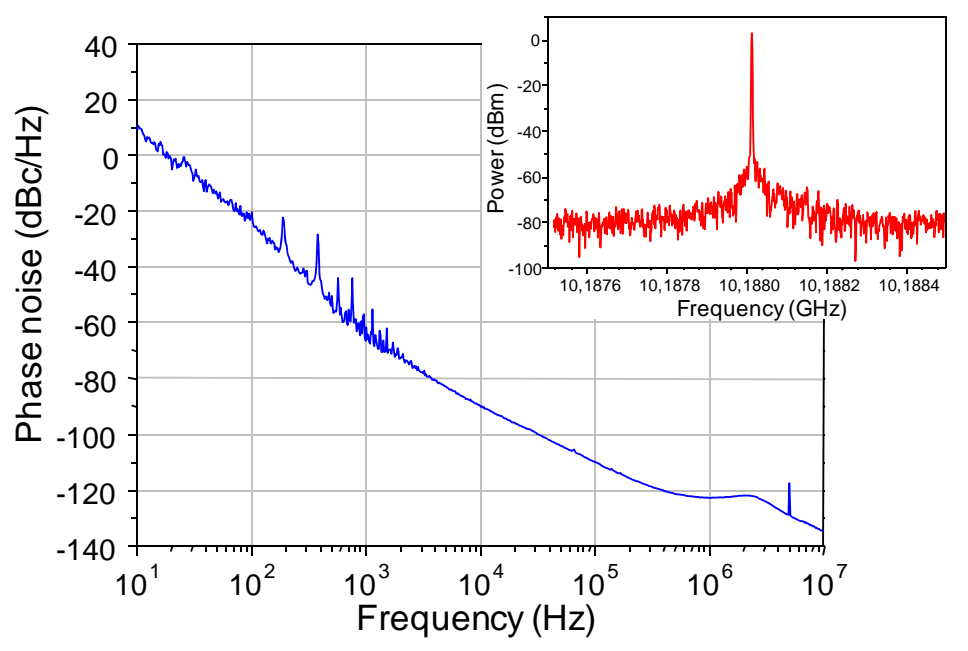

Figure 9. Phase noise measurement of the $10.2 \mathrm{GHz}$ OEO referenced on a fiber ring resonator

The OEO described above has been realized using a high spectral purity laser, to facilitate the lock onto the resonator. However, this system will be interesting for applications only if it could work on a (low size and low cost) semiconductor laser. Actually, this is possible with a lock technique which not only stabilizes the slow drift in frequency between the laser and the resonator, but also improves the laser linewidth. The goal is thus to realize an external cavity semiconductor laser referenced onto the ultra high Q resonator, then to use this resonator as a filter for the microwave signal. This is possible by increasing the bandwidth of the laser lock loop, either with the Pound Drever Hall technique or with another (faster) technique of laser stabilization. The case of optical locking should also be considered, as an efficient alternative to complex electronic locking of the laser (providing the feedback in phase of the signal towards the laser can be controlled). At this time, we are investigating the laser lock onto an optical frequency discriminator realized with an optical high Q resonator, as an alternative to the Pound Drever approach. The locking bandwidth is large enough to improve the laser spectral purity in this system. This is due to the choice of a feedback onto the laser bias current, and to a reduced length of the feedback. However, at this time of our study, the laser sprectrum improvement is not sufficient with this approach and we will need to improve the whole system to get a good performance with a semiconductor laser.

A key point is also to study the frequency stability of these oscillators versus any external perturbation, and particularly a temperature shift of the resonant ring or of the whole OEO system. We have studied experimentally the susceptibility to temperature variations of various optical fiber rings and OEOs based on these resonators. The resonators were placed in a temperature chamber, and their temperature was shifted from $20^{\circ} \mathrm{C}$ to $50^{\circ} \mathrm{C}$ or more. A shift of the whole optical comb of the resonator has been observed with the scan technique, but no significant variation of the ISL 
has been noticed, either by the optical scan technique or by the (more precise) microwave spectrum measurement technique described in 3.3. Actually, the comb is shifting but the frequency difference between the comb lines remains the same. The same experiment has been performed on a OEO, and the oscillation frequency was more sensitive to the fluctuations in the external RF loop than to the temperature controlled shift of the resonator. Between $20^{\circ} \mathrm{C}$ to $50^{\circ} \mathrm{C}$, no frequency shift of the OEO could be noticed, with a precision of $8 \mathrm{kHz}$ (resolution bandwidth during the measurement). Therefore, we believe that these oscillators, because they are based on the beat frequency between two (or more) resonator modes, will be exceptionally stable versus temperature when they will be assembled in a small volume.

Finally, the $\mathrm{Q}$ factor of our resonators is fixed by the finesse of the optical resonances. This means that the equivalent microwave $\mathrm{Q}$ factor in these systems increases proportionally to the microwave frequency (the $\mathrm{Q}$ factor is the ratio of the microwave frequency to the resonator half bandwidth, which is fixed in this case). This is a unique feature, which is diametrically opposed to what can be observed in conventional microwave resonators, and which should allow the development of high performance OEO systems in the millimetre wave frequency range (above $30 \mathrm{GHz}$ ). The first oscillator presented here has been designed at $10 \mathrm{GHz}$, because of the availability of both microwave and optical devices at this frequency in the laboratory. However, the same experiment could be performed at higher frequencies, with an improvement in Q proportional to the ratio in frequency. We could take benefit, as an example, of all the devices and techniques studied for radio over fiber systems at $60 \mathrm{GHz}$. The development of millimetre wave OEO will thus be one of the future challenges in OEO technology.

\section{CONCLUSION}

Optical resonators allow to reach really high quality factors in the microwave range, and even better quality factors in the millimetre wave range. This technology overcomes the limitations of conventional microwave technology for the development of high spectral purity microwave sources or filters. Among the resonators studied, whispering gallery modes resonators are potentially interesting for compact oscillators, if solutions for an efficient assembly can be found to propose integrated (or semi-integrated) coupling for these devices. On the other hand, fibered resonators, such as fibre ring resonators, are easier to integrate and use. These resonators are almost ready to replace in the optoelectronic oscillators technology the long delay lines which have been currently used up to now. However, the first results obtained with this approach, like the OEO presented in this paper, feature a phase noise which is not yet at the level expected for the applications foreseen for these systems. A huge amount of work is still going on to improve this noise performance, and also to realise compact systems based on low cost semiconductor lasers.

\section{ACKNOWLEDGEMENTS}

This study is performed in the frame of two on-going contracts, with CNES (french national space centre) and EDA (European Defense Agency) through ARAMOS program. We would like to thank P. Feron and S. Trebaol, from ENSSAT Lannion, for the joint work on resonators performed in the frame of these contracts.

\section{REFERENCES}

1. X.S. Yao, L. Maleki, “High frequency optical subcarrier generator," Electronics Letters, vol. 30, Issue 18, pp. 15251526, Sep. 1994.

2. X.S. Yao, D. Eliyahu, L. Maleki, "Progress in the optoelectronic oscillator - a ten year anniversary review," IEEE Microwave Theory and Tech. Symp. Digest, vol. 1, pp. 287-290, Jun. 2004.

3. P.H. Merrer, H. Brahimi, O. Llopis, "Optical techniques for microwave frequency stabilization : resonant versus delay line approaches and related modelling problems”, Proc. of the IEEE Int. Topical Meeting on Microwave Photonics, Gold Coast, Australia, Sept. 2008, pp. 146-149.

4. D. Eliyahu, D. Seidel, L. Maleki, "RF Amplitude and Phase-Noise Reduction of an Optical Link and an OptoElectronic Oscillator”, IEEE Trans. on Microwave Theory and Tech., Vol. 56, №. 2, Feb. 2008.

5. L. Maleki, X.S. Yao, J. Yu, V. Ilchenko, "New schemes for improved opto-electronic oscillator," Int. Topical Meeting on Microwave Photonics, MWP99, pp. 177, Nov. 1999. 
6. A.A. Savchenkov, V.S. Ilchenko, A.B. Matsko, L. Maleki, "Photonics frequency synthesis and control with whispering gallery mode microresonators", IEEE LEOS Newsletter vol. 17, pp. 22-23, 2003.

7. A. A. Savchenkov, E. Rubiola, A. B. Matsko, V. S. Ilchenko, L. Maleki, "Phase noise of whispering gallery photonic hyper-parametric microwave oscillators”, Optics Express, March 2008, Vol. 16, N. 6, pp. 4130-4144.

8. V. S. Ilchenko, J. Byrd, A. A. Savchenkov, A. B. Matsko, D. Seidel, L. Maleki, "Miniature Oscillators Based on Optical Whispering Gallery Mode Resonators”, Proc. of the 2008 IEEE Int. Freq. Control Symp., pp. 305-308.

9. X. S. Yao, L. Davis, L. Maleki, "Coupled optoelectronic oscillators for generating both RF signal and optical pulses”, Journal of Lightwave Technology, Vol. 18, Issue 1, Jan. 2000, pp. 73 - 78.

10. P.H. Merrer , A. Bouchier , H. Brahimi , O. Llopis , G. Cibiel, "High-Q optical resonators for stabilization of high spectral purity microwave oscillators” European Freq. and Time Forum and IEEE Int. Freq. Control Symp., April 2009, pp. 866-869

11. A.A. Savchenkov, A.B. Matsko, V.S. IIchenko, L. Maleki, "Optical Whispering Gallery Mode Resonators withQ > $10^{11}$ and $\mathrm{F}>10^{7}$, , Quantum Electronics and Laser Science Conference QELS '07, May 2007, pp. 1-2.

12. P.H. Merrer, O. Llopis, S. Bonnefont, P. Feron, G. Cibiel, “Microwave filtering using high Q optical resonators”, Proc. of the 2008 European Microwave Conference, Amsterdam, Oct. 2008, pp. 381-384.

13. A.B. Matsko, A.A. Savchenkov, N. Yu, L. Maleki, "Whispering-gallery-mode resonators as frequency references. I. Fundamental limitations”, J. Opt. Soc. Am. B, Vol. 24, No. 6, June 2007, pp. 1324-1335.

14. A.A. Savchenkov, A.B. Matsko, V.S. Ilchenko, N. Yu, L. Maleki, "Whispering-gallery-mode resonators as frequency references. II. Stabilization”, J. Opt. Soc. Am. B, Vol. 24, No. 12, December 2007, pp. 2988-2996.

15. P.H. Merrer, O. Llopis and G. Cibiel, "Laser stabilization on a fiber ring resonator and application to RF filtering," IEEE Photonics Tech. Letters, vol.20 (16), pp. 1399-1401, 2008.

16. Y. Dumeige, S. Trebaol, L. Ghisa, T.K.N. Nguyen, H. Tavernier, P. Feron, "Determination of coupling regime of high-Q resonators and optical gain of highly selective amplifiers," Journal of the Optical Society of America B 25(12), pp. 2073-2080 (2008).

17. D.B. Leeson, "A simple model of feedback oscillator noise spectrum," Proc. of the IEEE, Vol. 54, N², pp. 329-330, 1966.

18. H. Brahimi, H.L. Martinez-Reyes, P.H. Merrer, A. Bouchier, O. Llopis, “A CAD approach of microwave optical systems including noise performance”, Proc. of the 2009 European Microwave Conf., Oct. 2009, pp. 1642-1645. 